\title{
EDGAR WILLEMS
}

\section{NUEV AS IDEAS FILOSÓFICAS SOBRE LA MÚSICA Y SUS APLICACIONES PRACTICAS*}

Nuevas ideas ... quizás la afimación es osada e inclusive incorrecta pues ¿ acaso ya no se ha dicho todo sobre las leyes de la vida y sobre las de la Música? Pero cuando en la primavera las flores brotan otra vez, después del letargo invernal, acaso no decimos: ¡He allí las flores nuevas! La novedad consiste entonces en una nueva relación que existe entre una idea y la época, e inclusive entre una idea y su aplicación.

No nos detendremos entonces en el significado limitado de la palabra; las palabras siempre resultan insuficientes cuando se trata de hablar de la vida, y es precisamente sobre la vida musical que yo quisiera tratar aquí.

En efecto, hay vida y forma, cualidad y cantidad, arte y material sonoro, vida rítmica que es causa y formas rítmicas que son realmente los efectos, es píritu, técnica y formas de técnicas. Nosotros estamos siempre en presencia de dos elementos complementarios:

1) La forma, siempre visible, que cae fácilmente sobre nuestros sentidos, de la cual uno se ocupa demasiado y se preocupa con especial cuidado para desarrollar en el alumno.

2) La vida, el espíritu, tan a menudo ignorados incomprendidos.

Me propongo revisar, quizá de una manera demasiado condensada, algunos aspectos de la vida, as pectos que son idénticos en la música. Voy a hablar de la unidad de la vida, de su dualidad y del triple aspecto de la naturaleza humana.

De la unidad habría mucho qué decir; el tema es a la vez muy simple y demasiado complejo; así que tan solo diré algunas palabras: la unidad es el elemento principal hacia el cual se debe encauzar la obra del arte.

En la vida y en la naturaleza, una flor, un animal, un ser humano, repres entan siempre una unidad intrínseca. Lo mismo, la obra de arte contiene esa unidad. Unidad no solam ente técnica, de forma exterior, sino unidad de vida interior, que no siempre es fácil de revelar, y unidad entre las dos.

\footnotetext{
* Conferencia de EDGAR WLLLEMS, hecha en París, el 6 de enero de 1934, siendo profesor del Conservatorio de Ginebra, y bajo los auspicios de "La Música para todos". Edición revisada, 1966.
} 
En el caso de los grandes creadores, la unidad existe antes de la creación de la obra. Beethoven, refiriéndose a sí mismo, dice que antes de escribir una obra la concebía primero en su conjunto. Mozart dice que antes de escribir una composición la realizaba primero mentalmente.

Bach, en este punto, no se asemeja ni a Mozart ni a Beethoven.

Spitta, quien ha estudiado muy de cerca este asunto, afima que Bach realizaba de un golpe el conjunto de la obra y que nunca llegaba a suprimir un elemento o a invertirlo, lo que sí hacía Beethoven muy a menudo. Añadiremos en favor de Beethoven que, si Bach se contentaba la mayor parte del tiempo con formas conocidas y establecidas, Beethoven siempre estaba a la búsqueda de nuevas formas.

Determinar esta unidad es casi tan imposible como deteminar la vida; podemos sí a menudo presentirla, sentirla, podemos estudiar su carácter, sus diferentes aspectos. En muchos casos ese análisis es superficial y no concierne sino a la forma. Esta unidad exterior se constata fácilmente en la pintura, pues su materia contiene un elemento es table y material: el color. Entretanto el sonido, a la vez inmaterial y sometido al paso del tiempo, escapa al control inmediato de nuestros sentidos. Sin embargo, el músico profesional y el compositor saben reconocer esta unidad fomal, y aunque ella colabora en buena parte a la unidad espiritual de la obra, es apenas "el vestido".

Si analizamos esta unidad, nos enfrentamos en primer lugar a "dos polos opues tos y complementarios : la materia sonora y el espíritu artístico.

¿Toda manifestación, cósmica o humana, es en sí una interferencia entre dos fuentes de energía? ¿ No estamos siempre en presencia de una fuerza y de un elemento material (energía condensada)? Además, cada uno de estos dos polos escapa en parte a nuestras investigaciones, pues los dos nos enfrentan a lo desconocido, sea de las leyes de la materia o de las del espíritu.

El ser humano está influenciado por los dos polos; visto desde otro ángulo, podemos decir que es el resultado de la mutua reacción de esos dos polos, que represento con el siguiente es quema:

\section{SER HUMANO}

Desconocido de las leyes materiales Desconocido de las leyes es pirituales POLO MATERIAL POLO ESPIRITUAL

Desconocido de las leyes del sonido Desconocido de las leyes artísticas 
Hipotéticamente admitimos que lo desconocido de las leyes materiales y de las leyes espirituales se reúne en un todo: el Cosmos.

llustramos este esquema con una definición de la Música. Escojo una de las más simples y más concisas: La Música es el arte de los sonidos.

Aquí están los dos polos netamente representados: el arte, elemento espinitual, y el sonido, elemento material. Si profundizamos estos dos términos nos encontramos delante de los enigmas de la vida espiritual y material, pues ni el espíritu, ni la naturaleza del sonido nos han entregado todos sus secretos.

En efecto, "el arte" es la expresión de lo bello. Expresión es vida y la vida nos conduce a lo desconocido. Lo bello es un aspecto del todo, de la vida, de lo desconocido.

El sonido es una vibración de la materia vibración de fuerza, o energía, o vida - La materia se nos presenta, en un último análisis, bajo $\mathrm{cl}$ aspecto de moléculas, de átomos y de partículas: nuevamente vamos hacia lo desconocido.

Entre el polo de la materia y el del espíritu, se coloca la vida humana. Y esta vida la consideramos bajo un "triple as pecto". Digo triple y no quíntuple o séptuple, para no tomar sino el aspecto mas simple, el más inmediato, el más práctico para la comparación con la música.

En esta forma, partiendo del polo material vamos hacia el polo espiritual: la vida física, afectiva y mental; con la posibilidad, por ambas partes, de ir hacia el infinito, lo que nos permite añadir, si lo queremos, elementos inframateriales (vida prenatal), o elementos supraintelectuales (intuición o espiritualidad). Nos limitaremos a los tres aspectos principales, puesto que son los más accesibles, dado que nos expresamos diariamente a través dc cada uno de ellos.

Ahora estamos frente a esos tres elementos fundamentales de la música: frente a la vida física colocamos el elemento rítmico, la vida, la propulsión rítmica; frente a la afectividad, la emoción, el sentimiento, ponemos el elemento melódico; frente a la inteligencia, la vida mental, colocamos el elemento amónico.

VID A HUMANA

MATERIA

Vida fisiológica

SONIDO

Vida rítmica
Vida afectiva

Vida melódica

MÚSICA
ESPÍRITU

Vida mental

ARTE

Vida armónica 
Es de comprender que este esquema no es sino una introducción en un mundo que escapa a cualquier sistematización. Mundo donde el Ritmo, la Melodía y la Armonía tienen cada uno un triple as pecto físico, afectivo y mental, conservando su característica propia.

Lo que nos interesa son primordialmente las relaciones que hay entre los elementos constitutivos de cada uno de ellos, y en segundo lugar, la naturaleza profunda y misteriosa de esos elementos. Dichas relaciones las podemos observar yexperimentar.

Explicar en pocas palabras cómo se justifican estas relaciones, es bastante difícil. Sin embargo trataré de dar una idea general y sobre todo de resaltar la unidad viviente que liga estos diferentes elementos, y que se expresa por medio de dos polos: el sonido yel arte.

Una vez que se haya comprendido esta clasificación, su utilidad se hace evidente, pues permite darse cuenta de la naturaleza de las facultades humanas y de su expresión, en el dominio musical.

Muchos es critores, musicólogos o filósofos, hablando de los artistas, hacen de ellos dos retratos diferentes: el del Creador y el del Hombre.

Además, por otra parte, tratan de establecer las relaciones que pueden existir entre el artista y su obra. Ahora bien, estas relaciones son mucho más vivas, mucho más profundas de lo que se supone en general, y si alguna vez se llega a separar la obra del artista de su vida diaria, es porque uno se detiene demasiado en las apariencias y considera al artista bajo un ángulo personal que no permite ver la unidad intrínseca del Ser, su unidad propia.

Es indis cutible que en todo artista hay una unidad de vida profunda, que se nos puede escapar, pero que a la vez engloba sus cualidades humanas y su producción artística.

Esta unidad puede expresarse por contraste y la concibe fácilmente un artista cuya obra evidencia un ideal quizás más grande que lo que llega a realizar en su vida diaria. Pero esas dos manifestaciones, contradictorias a primera vista, parten no obstante de un fondo común.

Podemos entonces admitir la posibilidad de encontrar raíces comunes entre los principales elementos de la naturaleza humana y de su expresión, a través de la música.

El conocimiento profundo de los elementos fundamentales de la música, tomados en su esencia vital, nos permitirá comprender mejor la música occidental, nuestra música, y comprender con conocimiento de causa las producciones musicales orientales y negras. 
Si partimos de bases humanas, la música de cualquier raza o época se hace accesible. También seremos capaces de seguir las creaciones de nuestros contem poráneos que con frecuencia se anticipan a su época.

Nuestra sensibilidad ensanchada, liberada, y nuestra conciencia profunda dc las leyes naturales, nos permitirán distinguir lo falso de lo verdadero, las combinaciones intelectuales de aquellas dictadas por las leyes de la vida.

El conocimiento y la sensibilidad deben reaccionar libremente y sin estar entrabadas por una opinión tomada de antemano, sea por la época o por la raza. Las relaciones que se establecen pueden ayudarnos a lograr esta meta.

He creído encontrar, por los sistemas filosóficos, la solución de los problemas musicales. Desafortunadamente los filósofos parten muy a menudo de consideraciones metafísicas o intelectuales. Ahora bien, como músicos podemos partir de la música en sí misma, comprendida como una manifestación directa del ser humano. Todo elemento musical, cualquiera que sea, está en relación directa con el elemento humano que le ha dado nacimiento.

También es preciso colocar las fuentes de la Música en el ser humano, en un nivel de profundidad tal, que partiendo de ellas, se puede seguir su desarrollo y su expresión en todos los dominios, en todas las actividades artísticas.

Para anticiparnos a ciertas objeciones, que tienden a diferenciar las diversas manifestaciones artísticas, diremos que, en efecto, las distintas artes contienen elementos de expresión particulares, siempre y cuando estos elementos sean tributarios de un sentido diferente (tacto, vista, oído). Pero esos elementos no son sino los medios de expresión a través de los cuales los "impulsos vitales" se exteriorizan. Estos básicos impulsos vitales (físicos, afectivos, mentales 0 intuitivos) son comunes a todas las artes, aunque naturalmente en grados diferentes.

Durante largos años, estos cuestionamientos han sido objeto de mis estudios. Para resolverlos me he abstenido de la especialización. Fui en busca de bases humanas suficientemente amplias, para que pudieran servir como punto de partida a las actividades humanas superiores más diversas, tanto clásicas como modernas, conservadoras como evolucionistas. Habiendo practicado la mayor parte de las artes, lejos de ser un genio en ninguna de ellas, tengo la facilidad de manejar cierta información decisiva y poder demostrar hoy, cómo fuentes comunes alimentan las diferentes actividades humanas, y cómo raíces comunes les dan la fuerza para crecer y producir frutos.

Para determinar con precisión estos elementos, tomados desde su punto de partida, me veo forzado a recurrir a algunas abstracciones matemáticas 0 geométricas. 
Voy a hablar de cifras y de dimensiones.

Las cifras pueden ser consideradas bajo tres aspectos:

1) "cardinal" (uno, dos, tres. .

2) "ordinal" (primero, segundo, tercero...).

3) "filosófico" (unitario, dual, ternario ..

Las dimensiones también pueden ser consideradas bajo este triple as pecto. Se pueden representar las tres dimensiones por línea, superficie y volumen. Según el aspecto "cardinal", la superficie tiene más valor que la línea, y el volumen más que la superficie. Según el as pecto "ordinal", siendo la línea el primer elemento, tiene prioridad, dado que sin ella las otras no pueden existir. Para hablar del aspecto "filosófico" de las dimensiones es preciso una significación más profunda, pues se trata de tres dominios de vida y de conciencia (unidimensional, bidimensional, tridimensional, etc.) que tendrán entre ellos las mismas relaciones que los elementos geométricos; pero aquí la línea es considerada como una dirección, un movimiento, sobre el cual nada podemos quitar; la superficie, en cambio, nos permitirá extender un color; el cubo tendrá además un contenido.

Encontramos estas tres dimensiones de vida representados en los mundos vegetal, animal y humano.

Lo establecemos en el siguiente esquema:

$\begin{array}{lll}\text { Uno } & \text { Dos } & \text { Tres } \\ \text { Línea } & \text { Superficie } & \text { Volumen } \\ \text { Vida física } & \text { Vida afectiva } & \text { Vida intelectual } \\ \text { Vida ritmica } & \text { Vida melódica } & \text { Vida armónica } \\ \text { Mundo vegetal } & \text { Mundo animal } & \text { Mundo humano }\end{array}$

En primer lugar, este esquema nos pemite establecer relaciones que podrían ser consideradas corno inverosímiles en razón de la falta aparente de lazos entre los elementos de la música y esas abstracciones matemáticas o geométricas. Se puede dudar, inclusive, de su valor des de cl punto de vista práctico.

Ahora bien, creemos poder decir, al contrario, que este enunciado esquemático bien comprendido, puede ayudar en gran parte a resolver los problemas que se plantean en la actividad artística. Se trata sobre todo de ver en profundidad más allá de las formas que caen inmediatamente sobre los sentidos. Al igual que bajo los distintos ropajes bajo los aspectos infinitamente variados de los individuos, se esconde la misma estructura invisible. Asimismo, en el arte, leyes simples y profundas se mimetizan ante nues tros ojos.

Si volvemos a tomar el ejemplo de las tres cifras, aplicadas a la música, comprendemos que, si de una parte el "uno" contiene potencialmente el "dos", asimismo el "Ritmo", elemento primero en la música, contendrá también 
potencialmente la "Melodía", elemento segundo y se le dará nacimiento. De la misma manera, la melodía contendrá potencialmente, a la "Armonía", elemento tercero.

Sin tratar de sostener aquí esta afimación, nos limitaremos a llamar la atención sobre el hecho de que el ritmo sonoro, por sus elementos de duración y de intensidad, provoca fluctuaciones en la altura del sonido, introduciéndonos así en el dominio melódico. Igualmente, una melodía bien equilibrada contiene ya en su estructura los acordes destinados a sos tenerla amónicamente.

Si consideramos el problema bajo otro ángulo, estaremos en presencia de otras relaciones, igualmente verdaderas y profundas a pesar de su aparente simplicidad: el "dos" contiene al uno y el "tres" contiene a la vez al "dos" y al uno Transfiriendo estas relaciones a la música, diremos que la melodía contiene necesariamente al ritmo y que la armonía contiene a la melodía y al ritmo. Aunque no podemos extendemos mucho sobre cada uno de estos puntos para demostrar la evidencia, esperamos sin embargo que se acepte un enunciado tan simple en provecho de hacernos penetrar en la naturaleza íntima de los elementos del arte y de la vida. o que pueda servir de punto de partida, tanto desde el punto de vista artístico como pedagógico, psicológico o filosófico. También nos parece que la mayor parte de las divergencias de opinión en el dominio artístico, y particularmente en el musical, se deben a la ignorancia de estos principios elementales.

Aquí habría otra consideración fundamental: no hay solución de continuidad entre los diferentes dominios y no deben ser considerados como elementos separados los unos de los otros. Su interpretación impide cualquier demarcación precisa:

1) entre el ritmo y la melodía;

2) entre és ta y el acorde (que es de orden amónico).

He aquí un ejemplo para ilustrar el primer caso que concierne al ritmo y a la melodía: golpeamos un ritmo sobre un tambor, o sobre un objeto sonoro: tendremos un fenómeno de puro orden rítmico. Variamos la intensidad y tendremos sonidos de diferentes alturas.

Cantamos un ritmo sobre el mismo sonido; enseguida lo cantamos elevando ligeramente un sonido, un cuarto de tono, luego un semitono; continuamos luego modificando varios sonidos y estaremos en presencia de un fragmento melódico. ¿A partir de qué momento preciso entramos en contacto con el elemento melódico?

En lo que concierne al segundo caso, el que tiene relación con los dos elementos, melódico y armónico, tendremos también una graduación insensible si partimos del intervalo de octava (que es considerado como unísono) para demostrar en el acorde (unión de tres sonidos que se amonizan entre sí). Las 
diferentes etapas serán: la quinta y la cuarta, la tercera y la sexta. Estos dos últimos intervalos tienen ya un valor armónico (los sonidos resultantes nos lo demostrarán); en efecto, a estos dos sonidos viene a añadirse automáticamente, un tercer sonido que cada oído sensible puede descubrir. Este sonido es la quinta para el intervalo de tercera y la cuarta para el intervalo de sexta, formando as í en los dos casos un acorde sobreentendido. Agreguemos que las diferentes formas de polifonía son también grados intermediarios entre la melodía y la armonía.

Podemos establecer las mismas relaciones en lo que concierne a los elementos de la vida unidimensional (física), bidimensional (afectiva) y tridimensional (mental).

En este momento, dejamos de lado el elemento cuatridimensional, que completaría este conjunto, y nos haría sobrepasar los límites del tema que nos interesa y nos hemos impuesto.

Si se admite que la música puede tener bases universales y profundas y que un orden preside sus diferentes relaciones, entonces es inútil insistir sobre las numerosas consideraciones que de aquí se derivan. Nos contentaremos con dar algunas ilustraciones de los esquemas y algunos ejemplos prácticos, tomados en diferentes dominios.

Consideremos un instante el "toque del pianis ta". Iremos de los elementos más densos a los elementos más sutiles: primero está la mano; esta mano funciona; tocando el teclado adquiere una cierta ejecución, tacto. Estamos aquí en el dominio de los sentidos; el oído y la vista participan en el toque; la sensibilidad de los órganos se desarrolla y reacciona sobre nuestra afectividad. La imaginación musical, luego la memoria, se abren paso; gracias a la memoria, comparaciones y juicio colaboran al desarrollo de la conciencia musical. La inteligencia concreta al principio, llega a ser abstracta. La audición interior se desarrolla cada vez más y permite combinaciones de sonidos nuevos, creaciones: el pianista improvisa y compone.

Este conjunto, visto des de otro ángulo, puede perfectamente ser interpretado en sentido contrario, es decir del polo es piritual al polo material.

El cuadro puede invertirse; diría inclusive que podríamos rodarlo como se rueda una esfera: el orden y las relaciones pemanecen las mismas. Además si se dice que una composición musical parte del espíritu, de la inspiración y no de la materia, no se debe olvidar que esos elementos evolucionan entre dos polos, o que esos dos polos reaccionan continuamente el uno sobre el otro y la proyección puede venir sea de un polo o del otro, o de un elemento intermediario.

En efecto, la composición musical puede partir de la naturaleza del sonido, de su timbre, de una impulsión rítmica corporal, del amor por un sonido o un acorde, de una emoción, de un afecto o de un sentimiento, de una consideración intelectual, de una intuición o de una inspiración. Esto no quiere decir que uno de estos elementos baste para hacer una composición musical. La presencia de la 
mayor parte de estos elementos es simultánea, pero surge en un desorden aparente. Es un fenómeno musical como un juego de ecos; el espectador no ve a primera vista sino una mezcla de la cual él no conoce el secreto; los intérpretes se dan perfecta cuenta del orden que preside la mezcla de las piezas, y el buen intérprete conoce a la vez el orden del sonido tocado y el que toca su compañero dentro de un conjunto. Igualmente el músico puede conocer todos los elementos de la música y el orden que presiden sus diferentes combinaciones.

La "naturaleza del sonido" nos ofrece una ilustración del segundo esquema que concieme a los tres dominios.

En efecto, el sonido tiene tres cualidades: la intensidad, la altura y el timbre. Hay dos relaciones estrechas entre la intensidad y el ritmo, entre la altura y la melodía, y entre el timbre y la amonía (pues el timbre es el resultado de un conjunto de sonidos armónicos que se unen al sonido fundamental). Gracias a aparatos sencillos, actualmente se puede estudiar de cerca la naturaleza del sonido. Los oscilógrafos y los analizadores amónicos transforman el sonido en gráficos y permiten así hacer los cálculos más precisos en lo que concierne a la amplitud de la vibración (intensidad), su frecuencia (altura) y su forma particular (timbre).

Tendremos cuidado en la aplicación, si admitimos las bases y las relaciones enunciadas, en lo que concierne al "ritmo", para trabajarlo física o muscularmente. Si componemos o improvisamos, partiremos a menudo del dinamismo corporal efectivo o imaginado; la imaginación plástica puede reemplazar al movimiento efectivo. En la enseñanza tendremos en cuenta la naturaleza física del ritmo (que por extensión puede llegar a ser ritmo emotivo o mental) y pediremos al alumno una colaboración física que consistirá, al menos, en marcar el compás con todo el brazo, de manera que haya contacto entre la mano que marca y los pulmones que son, con el corazón, el centro dinámico del cuerpo (el brazo y el hombro no serán inmovilizados).

En muchos casos, si un alumno improvisa mal, es porque le falta impulso rítmico (corporal); un paso hacia atrás se impone, y los ejercicios de improvisación rítmica deberán preceder la improvisación melódica. Bien sabemos que muchos profesores parten de bases mentales para la improvisación, como también para la música en general. Inclusive el ritmo se trabaja con la ayuda de los valores intelectuales: blancas, negras, corcheas, etc. pero de esta manera ¿dónde está el lazo de unión con la vida?, ¿o el lazo con el ser humano de nervio y músculo?, ¿o el de la emoción, que a menudo se traduce físicamente (rítmicamente)?

Para la melodía, como parte del ritmo, la inteligencia no basta; no es la base, el punto de partida.

El elemento melódico es de segundo dominio, y por supuesto el de la sensibilidad; y es con ella y a través de ella, que el músico lo expresará. Es también a la sensibilidad que el pedagogo recurrirá cuando el alumno esté 
melancólicamente deficiente. Cuántas veces he tenido que destacar la intrusión del pensamiento, en los alumnos principiantes, cuando se trata de cantar una frase de ocho o dieciséis compases. Obstruido por la inteligencia, el alumno no llega a cantar sino sucesiones de sonidos desprovistos de vida interior, porque la inteligencia, en este caso, se encarga del dominio que no es suyo, el que inhibiendo la frase, surja instintivamente suave yarmoniosa.

Bien sabemos que cualquier sentimiento, o emoción, puede ser traducido a música. ¿ Es sólo privilegio de los grandes maestros el vivir y expresar su sensibilidad a través de la música? Por intuición y muchas veces a pesar de la enseñanza recibida, ellos han establecido los lazos vivos de unión entre o su arte y la naturaleza humana.

¡Cuántos alumnos han comenzado jóvenes la música, con alegría y entusiasmo! Esa alegría ¿acaso ha aumentado, se ha enriquecido de experiencias en el curso del trabajo? ¿Ese entusiasmo se ha incrementado con el descubrimiento progresivo de lo espléndido de la vida? O bien, ¿ el alumno se ha confiado tan sólo al intelecto que promete darle lo que él no posee en sí mismo?

¿Es tan difícil conservar en la música los lazos que la unen con la vida?

En lo que concieme al tercer elemento, "la armonía”, podemos as ignar un lugar preponderante al intelecto y a la inteligencia. No se trata, en efecto de escuchar varios sonidos a la vez, lo que según los filósofos se efectúa únicamente gracias al cerebro, capaz de análisis y de síntesis. En la amonía estamos precisamente en presencia de la capacidad de síntesis y de abstracción.

Cuando hablamos por ejemplo del acorde de $7 a$ sobre el $\mathrm{V}$ grado, estamos en presencia de una unidad en la cual el sentido auditivo no puede tomar conciencia sino nota por nota. Otra cosa es entonces el acorde realizado auditivamente, por ejemplo: sol- sí- re- fa- y el pensamiento del acorde de 7 a sobre $\mathrm{V}$ grado (recordemos que el elemento armónico encierra necesariamente el elemento melódico). En la práctica se cometería un error capital queriendo obtener de un alumno o de sí mismo, un trabajo armónico por sola sensibilidad. En lo que concieme a la armonía propiamente dicha, la inteligencia juega un gran papel, diríamos que un papel de primer plano. En la obra de arte, armonía, melodía y ritmo se fusionan en una nueva unidad.

Tomo un último ejemplo en el "desarrollo auditivo". Aquí también los esquemas son aplicables en su complejidad y en su simplicidad. En la práctica de la enseñanza de la audición, estamos en presencia de diversos elementos, en los cuales encontramos el mismo orden y las mismas relaciones que en los de la música en general o en los elementos de la naturaleza humana.

Estos son: el órgano auditivo, el oído (polo material); el funcionamiento del oído (vida física); la sensibilidad afectiva (vida afectiva); la conciencia auditiva (vida mental); la audición interior con sus posibilidades de creación artística (polo espiritual). 
Cada uno de estos elementos puede ser el objeto de nuestra atención. El oído como "órgano", es poco modificable; en ciertos casos (enfemedad, sordera parcial), necesita de cuidados médicos. Depende del estado general del cuerpo. La vida del oído, su receptividad al sonido, se pueden aumentar con educación. Los músicos natos tienen por instinto una gran actividad auditiva. Los músicos por educación son a menudo demasiado cerebrales y muy poco sensoriales: en muchos de esos casos se impone una reeducación.

Podemos darnos cuenta del grado de sensibilidad física del oído por medio de un audiómetro; personalmente, utilizo un pequeño armonio para este fin; es un aparato que yo mismo he fabricado, y que contiene cincuentavos, centésimos y doscientavos de un tono. (Nunca he logrado obtener un ochocientavo de tono, lo cual no es límite, pues el sonido es divisible hasta el infinito).

No faltan los medios prácticos para aumentar la sensibilidad del oído. Citemos tan sólo dos en los cuales uno no piensa muy a menudo:

1) escuchar los ruidos de la naturaleza;

2) escuchar el sonido con un buen nivel de flexibilidad armónica, de manera que se puedan escuchar los sonidos amónicos y los sonidos resultantes (a menudo el pensamiento nos lo impide), sonidos que han tenido una gran importancia en la evolución musical.

En el dominio de la "sensibilidad afectiva auditiva", me gustaría llamar la atención sobre la relación que hay entre esta sensibilidad y los intervalos melódicos, no solamente como los encontramos en el piano, sino como los podemos conocer cuando logramos destrezas con el espacio intratonal. Melódicamente, en efecto, un re sostenido es parecido a un mi bemol. Por necesidades amónicas y también por necesidades prácticas para el instrumento, el teclado ha sido temperado. Por este hecho se le ha quitado a la melodía una de sus prerrogativas, que es la justeza natural, única capaz de expresar con exactitud ciertas emociones. Todos los cantantes, todos los músicos que tocan un instrumento de cuerdas, sin excluir las maderas e inclusive los cobres, deberían utilizar la justeza natural que les da una superioridad indiscutible, desde el punto de vista de la sensibilidad, sobre los instrumentos de teclado temperado.

Se puede ir más lejos, inclusive, diciendo que la justeza natural no está en el límite de nuestros medios, artísticamente hablando. Pues a menudo se trata no tanto de expresar una cosa tal cual es, como en las otras artes, sino de cómo deseamos que ella sea. En muchos casos, por ejemplo en el intervalo do- $m i$ bemol, se puede tomar el mi bemol de lo que sería hablando de justeza natural. Se puede hacer cuando se quiere acentuar el dinamismo de línea melódica. Este dinamismo es de una importancia capital en el arte, y hace que esta nota tienda a descender, y no se puede lograr un dominio con la práctica. 
En ocasiones, el nombre de la nota es a menudo un obstáculo para el desarrollo de la sensibilidad.

Con el intervalo amónico y los acordes entramos en el dominio de la "inteligencia auditiva". El oído, por sus ramificaciones, continúa su trabajo en el cerebro, que es el único capaz de hacer una síntes is consciente o inconsciente. Aquí el nombre de la nota es bienvenido; en muchos casos es absolutamente necesaria la toma de conciencia del dominio sonoro.

Cada intervalo y cada acorde serán objeto de un estudio profundo: son los primeros elementos que constituyen la música amónica. Se trata entonces de reconocer por la audición, cada uno de los intervalos y cada uno (le los acordes (inclusive sin el recurso de los nombres de las notas, sino con la sola conciencia del sonido).

He inventado un pequeño aparato llamado "el audiocultor", para el ejercicio de la audición de intervalos y acordes, ya que para la audición es preciso realizar prácticas entre dos personas: una da los acordes para adivinarlos y la otra los reconoce; este aparato, que consiste en un pequeño dispositivo que se coloca sobre el piano, y una serie de tablitas, que se activan por una simple presión, permiten producir los intervalos y los acordes para adivinar; en esta forma también se puede trabajar solo ${ }^{1}$

La última etapa del desarrollo auditivo es la audición interior. Aquí las sensibilidades física y afectiva se reúnen con la conciencia musical y se ponen al servicio de la creación artística musical, que puede sobrepasar las posibilidades de la inteligencia. Entramos en el dominio de lo desconocido, inmaterial, intuitivo o espiritual.

Para terminar, quisiera responder a la objeción que se hace a menudo sobre la intervención de la conciencia en el arte. Se dice: "No se hace necesario saber el por qué de las cosas. Cantidad de artistas han hecho sus obras de arte sin saber como.

Evidentemente, muchas personas hacen cosas sin saber cómo, e inclusive lo hacen sin saber lo que hacen. Esta conciencia no tiene ningún valor en si misma, y no es una caracterís tica de los grandes artistas; no adquiere valor sino después de haber pasado por el trabajo intelectual, para llegar a ser supraintelectual, a menos que sea intuitiva, lo cual demanda una madurez humana, o una receptividad es pecial.

Realmente, en arte la sensibilidad sobrepasa a menudo la conciencia; también es cierto que es ta última, siguiendo un impulso interior, trate de consolidarse cada

\footnotetext{
${ }^{1}$ Nota de la traductora: se debe recordar que esta conferencia fue escrita en 1934 cuando no existían las grabadoras ni los computadores, que hoy en día (1992) han subsanado completamente este vacío.
} 
vez más. La evolución del arte está íntimamente ligada al desarrollo de la conciencia humana, dirigida hacia la conciencia de la belleza.

De esta manera, el trabajo musical se convierte cada vez más en una parte intrínseca de nosotros mismos. Se integrará a nuestro ser con nuestra vida diaria. Será realmente nuestra razón de existir. Comprenderemos y amaremos mejor a los grandes maestros, pues estaremos más cerca de ellos. Partiendo de las mismas fuentes, a pesar de nuestros débiles medios, comprenderemos mejor su lenguaje, inclusive sus almas que, aunque lejanas, nos guían hacia la belleza eterna. 